\title{
Lipid nanobubbles as an ultrasound-triggered artesunate delivery system for imaging-guided, tumor-targeted chemotherapy
}

This article was published in the following Dove Medical Press journal:

OncoTargets and Therapy

\author{
Shuang Gao'** \\ Xiaohui Cheng ${ }^{2, *}$ \\ Jinhua $\mathrm{Li}^{3}$ \\ 'Ultrasound Department, Guilin \\ People's Hospital, Guilin, Guangxi \\ 541002, China; ${ }^{2}$ Department of \\ Clinical Pharmacy, Daqing Oilfield \\ General Hospital, Daqing, Heilongjiang \\ 163000, China; ${ }^{3}$ Traditional Chinese \\ Medicine Department, Gansu \\ Provincial Hospital, Lanzhou, Gansu \\ 730000, China \\ *These authors contributed equally \\ to this work
}

Purpose: Herein, this study is to prepare folic acid (FA)-conjugated lipid nanobubbles (NBs) that highly load artesunate (Arte; FA-ALNBs), as an ultrasound (US)-triggered Arte delivery system for imaging-guided, tumor-targeted chemotherapy.

Materials and methods: The morphology, size, zeta potential, and stability of the FA-ALNBs were detected by optical microscopy and dynamic light scattering analysis. The cellular uptake of the FA-ALNBs was evaluated by confocal laser scanning microscopy and flow cytometry. Results: The FA-ALNBs showed uniform spheroidal structure, with $781.2 \pm 5.3 \mathrm{~nm}$ in average diameter, great physiological stability, and $\sim 91.9 \% \pm 1.1 \%$ encapsulation efficiency of Arte. Using focused US, about $36.1 \% \pm 2.5 \%$ of the entrapped Arte was trigger-released from the FA-ALNBs. Owing to the US contrast property, FA-ALNBs showed an enhanced US signal in vitro when using an ultrasonic diagnostic apparatus with a 1-MHz linear transducer. Due to the FA receptor-mediated endocytosis effect, FA-ALNBs can be efficiently internalized by cells, showing an uptake ratio of about $56.4 \% \pm 3.1 \%$. FA-ALNBs showed an enhanced, dose-dependent cell-killing ability, while FA-ALNBs plus US irradiation exhibited a stronger anticancer effect in vitro. Post intravenous injection into tumor-bearing mice, FA-ALNBs showed an enhanced US contrast effect with increase in time, indicating the increasing accumulation of FA-ALNBs in tumor tissue, which peaked at 4 hours post injection. Focused US irradiation was conducted on the tumor region at 4 hours post injection of FA-ALNBs, which showed a greater tumor suppression effect after 30 days of treatment compared with all other treatment groups. Moreover, FA-ALNBs showed negligible systemic toxicity in vivo.

Conclusion: This versatile US-triggered drug delivery system with great anticancer efficacy was assessed both in vitro and in vivo, revealing great potential as a cancer theranostic agent for future application.

Keywords: nanobubbles, artesunate, ultrasound imaging, ultrasound-triggered drug release, cancer theranostics

\section{Introduction}

In recent years, breast cancer has attracted increasing attention due to its high morbidity and mortality in women. ${ }^{1,2}$ As breast cancer presents with high proliferation, invasion, and metastasis, affected patients have low disease-free survival and overall survival rates. ${ }^{3}$ Traditional therapeutic methods for breast cancer include surgery, chemotherapy, radiation, and hormonal therapy. ${ }^{1}$ Among these, chemotherapy is widely used and generally accepted by the patients.

Although various synthetic anticancer drugs have shown significant therapeutic effects, their adverse side effects also threaten human health. In recent decades,
Traditional Chinese Medicin Department, Gansu Provincial Hospital No. 204, Donggang West Road, Chengguan District, Lanzhou, Gansu Province 730000 , China

Tel +869318281840

Email maxli_0613@foxmail.com 
A

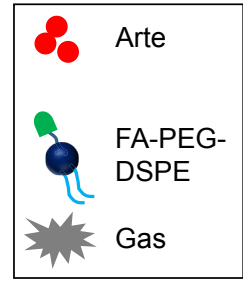

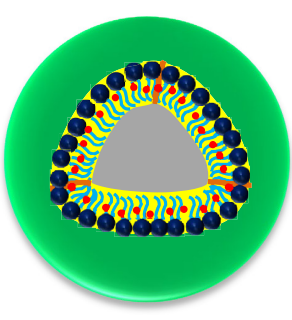

FA-ALNBs
B

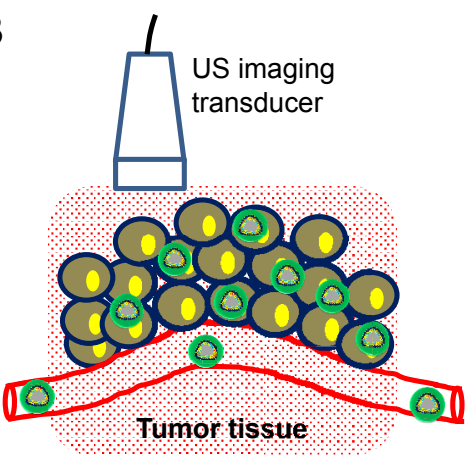

C

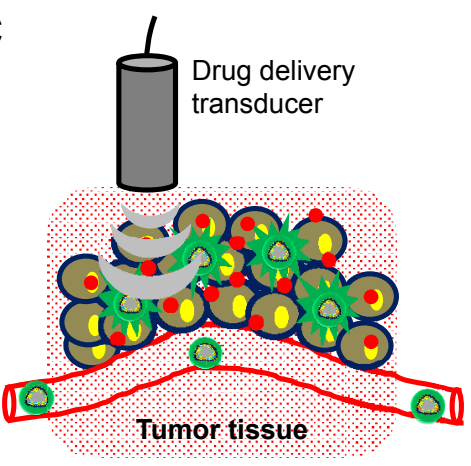

Figure I Synthesis and application schematic.

Notes: (A) Formation of FA-ALNBs. (B) Tumor US imaging. (C) US-triggered drug release for tumor therapy application.

Abbreviations: Arte, artesunate; DSPE, I,2-distearoyl-sn-glycerol-3- phosphoethanolamine; FA-ALNB, folic acid conjugated lipid NBs that highly load Arte; NBs, nanobubbles; PEG, polyethylene glycol; US, ultrasound.

plant extracts have provided a better alternative, with minimal side effects. ${ }^{4,5}$ Artesunate (Arte), a natural drug extracted from Artemisia аппиа, has been widely applied as an antimalarial agent over the last few decades. ${ }^{6-8}$ Arte is recommended by the WHO due to its good clinical efficacy and tolerability. ${ }^{9}$ The efficacy of Arte in the treatment of various cancers, such as cancer of the liver, lung, and breast, has also been demonstrated in the recent decades..$^{10-12}$ However, poor water solubility and low bioavailability of Arte limit its extensive clinical application. ${ }^{11}$ Various types of nano-carriers are widely used as delivery systems to increase the solubility and sustained release of Arte. For instance, Zhang et al designed novel Arte-loaded BSA nanoparticles to enhance the antitumor effects of Arte. ${ }^{9}$ Pang et al prepared Arte-modified nano-graphene oxide for chemophotothermal cancer therapy. ${ }^{11}$ Nevertheless, novel carriers are still required to improve the efficacy of Arte.

Nano- or microbubbles (NBs or MBs) and small air- or gas-filled nanospheres are important drug delivery vehicles, as they can stably transport drugs in the blood circulation..$^{13,14}$ Moreover, it was found that these bubbles exhibit alternate growing and shrinking under an external ultrasound (US) field. ${ }^{15}$ By increasing US frequency and pulse length, these bubbles could be destroyed, extending the application of NBs or MBs in site-specific drug delivery. ${ }^{16}$ In addition, the controllable drug release could enhance efficacy, while reducing undesirable side effects. Furthermore, due to their acoustic properties, NBs or MBs could act as US contrast agents for US imaging to guide drug delivery and tumor therapy. ${ }^{17-20}$ For example, Zhu et al developed a kind of MBs loaded with paclitaxel for US-triggered imaging-guided drug delivery, to inhibit vascular reconstruction. ${ }^{19} \mathrm{Lv}$ et al prepared folic acid (FA)-functionalized mesoporous silica nanoparticle-loaded
MBs via US-triggered destruction for enhanced targeted tumor therapy. ${ }^{15}$

Herein, we report tumor-targeting molecule FAconjugated lipid NBs that highly load Arte (FA-ALNBs), as a multifunctional platform for tumor theranostics (Figure 1). The morphology, size, and stability of the resulted FAALNBs were characterized using optical microscopy and dynamic light scattering (DLS) analysis. Under US irradiation, the FA-ALNBs not only enhanced the US contrast but also effectively trigger-released Arte. The cellular uptake of FA-ALNBs was detected both by confocal fluorescence microscopy and flow cytometry via labeling fluorescent dye to the NBs. The cytotoxicity of the FA-ALNBs with or without US irradiation was evaluated by a classic cell counting kit-8 (CCK-8) assay. As an US contrast agent, US imaging capacity was used to monitor the tumor accumulation of FA-ALNBs. The targeted anticancer efficacy of FA-ALNBs with or without US irradiation was then demonstrated both in vitro and in vivo. The obtained FA-ALNBs, which show great biocompatibility in vitro and in vivo, would be potential tumor theranostic agents for future nanomedicine applications.

\section{Materials and methods Materials}

Disaturated phosphatidylcholine (DSPC), 1,2-distearoyl-snglycerol-3-phosphoethanolamine-N-(folate [polyethylene glycol]-2000) (DSPE-PEG 2000 -FA), and 1,2-distearoylsn-glycero-3-phosphoethanolamine-N-(carboxy [polyethylene glycol]-2000) were supplied by Ponsure Biotechnology (Shanghai, China). Arte was purchased from Chongqing Huali Konggu Co., Ltd. (Chongqing, China) with purity $\geq 99.0 \%$. Perfluoropropane $\left(\mathrm{C}_{3} \mathrm{~F}_{8}\right)$ and all other chemicals were obtained from Sigma-Aldrich Co. (St Louis, MO, USA). 


\section{Preparation and characterizations of FA-ALNBs}

According to the previous reports, ${ }^{21}$ the DSPC and DSPE$\mathrm{PEG}_{2000}-\mathrm{FA}$ (4:1 molar ratios) were mixed with Arte (1 mg/mL, dissolved in dimethyl sulfoxide [DMSO]). The mixture was then dissolved in chloroform. Afterward, the chloroform was removed under nitrogen flow at room temperature. The dried mixture was hydrated in $5 \mathrm{~mL}$ PBS at $60^{\circ} \mathrm{C}$, and then was added to $\mathrm{C}_{3} \mathrm{~F}_{8}$. Next, the mixture was processed by mechanical vibration for 80 seconds. The unloaded Arte was eliminated via dialysis in deionized water for 6 hours, resulting in the Arte-encapsulated and FA-conjugated NBs (FA-ALNBs). As contrast, the FA-ALNBs without FA (ALNBs) and FA-ALNBs without Arte (FA-LNBs) were also prepared according to the method mentioned before.

\section{Characterizations}

The morphology, size, zeta potential, and stability of the FA-ALNBs were detected by optical microscopy (Olympus Corporation, Tokyo, Japan), transmission electron microscopy (TEM, SU8010; Hitachi Ltd., Tokyo, Japan), and DLS analysis using a Zetasizer Nano ZS system (Malvern Instruments, Malvern, UK). The cellular uptake of the FA-ALNBs was evaluated by confocal laser scanning microscopy (LEXT OLS4100; Olympus) and flow cytometry (FACSCanto II; $\mathrm{BD}$, Bedford, MA, USA). The absorption spectra of the nanoparticles were detected on a Lambda 35 UV-vis spectrometer (PerkinElmer Inc., Waltham, MA, USA).

\section{Arte encapsulation efficiency (EE) and release}

The Arte concentration was detected by a UV-vis spectrometer at Arte absorption peak wavelength $(280 \mathrm{~nm})$. The Arte EE was calculated as described in the following equation:

$$
\mathrm{EE}(\%)=\frac{\text { Arte }_{\text {total }}-\text { Arte }_{\text {unloaded }}}{\text { Arte }_{\text {total }}} \times 100 \%
$$

In addition, the cumulative release efficiency (CRE) of Arte with or without US irradiation was evaluated. A total of $1 \mathrm{~mL}$ of FA-ALNBs was placed in EP tube and sealed with parafilm. After filling up by ultrasonic coupling agent, the EP tube was placed onto the surface of the US transducer. After 1 minute US irradiation, these tubes were processed by centrifugation with $10,000 \mathrm{~g}$ for 30 minutes. The US irradiation was generated by a $1 \mathrm{MHz}$ transducer with $1 \mathrm{MPa}$ US intensity. The supernatant was collected and extracted by $\mathrm{CHCl}_{3}$ and the absorbance at $292 \mathrm{~nm}$ wavelength was detected. The CRE was calculated as described in the following equation:

$$
\operatorname{CRE}(\%)=\frac{\text { Arte }_{\text {free }}}{\text { Arte }_{\text {total }}} \times 100 \%
$$

\section{Cell culture and cellular uptake}

Murine mammary adenocarcinoma cells (4T1 cell) were provided by American Type Culture Collection (Manassas, VA, USA). The 4T1 cells were cultured in Roswell Park Memorial Institute (RPMI)-1640 (pH 7.2) supplemented with $10 \%$ FBS and $1 \%$ penicillin-streptomycin solution, and maintained in a humidified atmosphere of $5 \% \mathrm{CO}_{2}$ in air at $37^{\circ} \mathrm{C}$.

For cellular uptake, fluorescein isothiocyanate (FITC) was applied to label the ALNBs and FA-ALNBs. A total of $1 \mathrm{mg}$ FITC was dissolved in $1 \mathrm{~mL}$ DMSO and then mixed with the NBs with slight stir for 10 minutes. Afterward, the mixture was dialyzed in deionized water for 12 hours to remove the free FITC, resulting in FITC-labeled NBs. 4T1 cells were cultured for 24 hours in FA-free medium, and then free FITC, ALNBs, FA-ALNBs, and FA-ALNBs + FA (with same FITC concentration) were incubated with the 4T1 cells for 6 hours. The treated cells were washed with PBS three times and fixed with 4\% paraformaldehyde. After that, the cells were stained with $0.2 \mathrm{mg} / \mathrm{mL}$ of DAPI solution for 10 minutes. The cells were divided into two sections. One section cells were imaged by confocal laser scanning microscopy to capture the fluorescence images. And the other section cells were analyzed by flow cytometer to detect the fluorescence signal (F) of the cells. The calculation equation was as follows: Uptake ratio $(\%)=\frac{\mathrm{F}_{\text {FITC }}}{\mathrm{F}_{\mathrm{DAPI}}} \times 100 \%$, where $\mathrm{F}_{\mathrm{FITC}}$ was the cellular fluorescence value of FITC or FITC-labeled NBs treated cells, and $F_{D A P I}$ was the cellular fluorescence value of DAPI-treated cells.

\section{In vitro hemolysis test}

For hemolysis test of FA-ALNBs, $1 \mathrm{~mL}$ whole blood was collected from a healthy mouse. Through centrifugation, the red blood cells (RBCs) were separated. FA-ALNBs at different concentrations in PBS were incubated with RBCs. After 1 hour incubation at $37^{\circ} \mathrm{C}$, the treated $\mathrm{RBCs}$ were centrifuged at 10,000 rpm for 1 minute. The absorbance of the supernatants was detected by UV-vis spectrophotometer at $541 \mathrm{~nm}$. 
The hemolytic percent (HP) was calculated according to the following equation:

$$
H P(\%)=\frac{A_{t}-A_{n c}}{A_{p c}-A_{n c}} \times 100 \%
$$

where $A_{t}, A_{p c}$, and $A_{n c}$ are the absorbance value of the supernatant of the test sample, water-treated sample (positive control), and PBS-treated sample (negative control), respectively.

\section{In vitro anticancer efficacy}

First, FA-LNBs with the concentrations of 0, 50, 100, 250, and $500 \mu \mathrm{g} / \mathrm{mL}$ were cultured with $4 \mathrm{~T} 1$ cells. After 24 hours treatment, the cells were treated with RPMI-1640 media containing $10 \%$ CCK-8 for 20 minutes in an incubator. The absorbance of the cells at $450 \mathrm{~nm}$ wavelength was detected by a Multimode Plate Reader (EnVision; PerkinElmer) to characterize the cell viability. In addition, Arte, ALNBs, and FA-ALNBs with the same concentrations of Arte $(0,10,20,30$, and $40 \mu \mathrm{g} / \mathrm{mL})$ with or without 1 minute US irradiation were treated with 4T1 cells for 6 hours. After that, the cells were incubated for further 24 hours. The cell viability was also evaluated by CCK- 8 assay.

\section{Animal model}

Female Balb/c nude mice (5 weeks old) were provided from Vital River Laboratory Animal Technology Co., Ltd. (Beijing, China). The tumor model was established by subcutaneous injection of $150 \mu \mathrm{L}$ PBS containing $5 \times 10^{6} 4 \mathrm{~T} 1$ cells into the right back regions of the mice. After about 7 days, the tumor growth was observed by naked eye and the tumor volume was detected using a caliper. The tumor volume was calculated according to the following equation: Volume $=($ length $) \times(\text { width })^{2} / 2$. The animals' experiments were conducted in accordance with the Guidance Suggestions for the Care and Use of Laboratory Animals. This study, involving all animal experimental procedures was approved by the Ethics Committee of Traditional Chinese Medicine Department, Gansu Provincial Hospital.

\section{In vivo US imaging}

First, FA-ALNBs were dispersed in PBS and then mixed with agarose gel to form a pie-shaped phantom. The US images were captured by using a US imaging system from the horizontal section of the phantom. Furthermore, previous to and after 2, 4, and 6 hours intravenous injection of
FA-ALNBs into tumor-bearing mice, the US images of the tumor regions were also captured.

\section{In vivo anticancer efficacy}

When the tumor volume of the mice reached $60 \mathrm{~mm}^{3}$, 4T1 tumor-bearing mice were randomly divided into seven groups $(n=5)$ : group 1: saline (control); group 2: US only; group 3: free Arte; group 4: ALNBs; group 5: ALNBs plus US irradiation (ALNBs + US); group 6: FA-ALNBs; and group 7: FA-ALNBs plus US irradiation (FA-ALNBs + US). Different samples with equivalent Arte dose of $6 \mathrm{mg} \mathrm{kg}^{-1}$ were intravenously injected into the tumor-bearing mice. After 4 hours of injection, US wave was irradiated on the tumor region. An UTG-1025 US transducer with working intensity of $2 \mathrm{~W} / \mathrm{cm}^{2}$ and $1 \mathrm{MHz}$ frequency (Chongqing) was applied for in vivo treatment. During the course of treatment, the tumor volume and body weight of the mice were monitored every 3 days. The relative tumor volumes were calculated according to the following equation: relative tumor volume $=\mathrm{V} / \mathrm{V}_{0}$, where $\mathrm{V}_{0}$ represents the initial tumor volume.

\section{In vivo biosafety evaluation}

After the treatment, the mice were sacrificed to collect the major organs, including heart, liver, spleens, lung, and kidneys. These organs were successively fixed in 4\% paraformaldehyde, embedded in paraffin, dehydrated, and sliced into $5.0 \mu \mathrm{m}$ sections. According to the recommended protocols, the sections were stained with H\&E and examined by a light microscope. In addition, $150 \mu \mathrm{L}$ of FA-ALNBs at a dose of $10 \mathrm{mg} / \mathrm{kg}$ was intravenously injected into healthy mice $(\mathrm{n}=5)$. Before and after 3 and 7 days, the whole blood was collected for complete blood counts evaluations.

\section{Statistical analysis}

Data were shown as mean $\pm \mathrm{SD}$. Two-tailed Student's $t$-test was used to analyze the statistical significance of two groups. The differences were considered significant for $* P<0.05$ and highly significant for $* * P<0.01$.

\section{Results and discussion Preparation and characterization of FA-ALNBs}

The prepared FA-ALNBs were uniformly distributed, as seen on light microscopy and TEM images (Figure 2A). The size of the FA-ALNBs varied from $650 \mathrm{~nm}$ to $910 \mathrm{~nm}$, with an average particle diameter of $781.2 \pm 5.3 \mathrm{~nm}$ (Figure $2 \mathrm{~B}$ ). The zeta potential of the FA-ALNBs was detected to be $-31.2 \pm 1.2 \mathrm{mV}$ (Figure $2 \mathrm{C}$ ). The stability test revealed a 
A

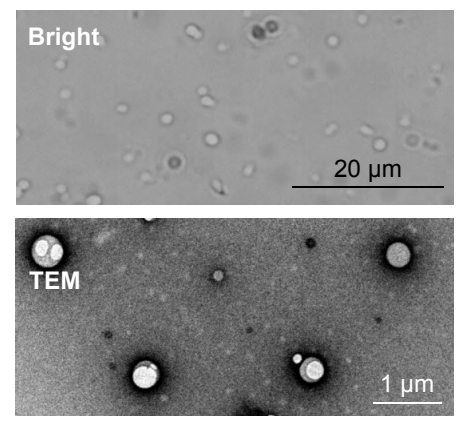

D

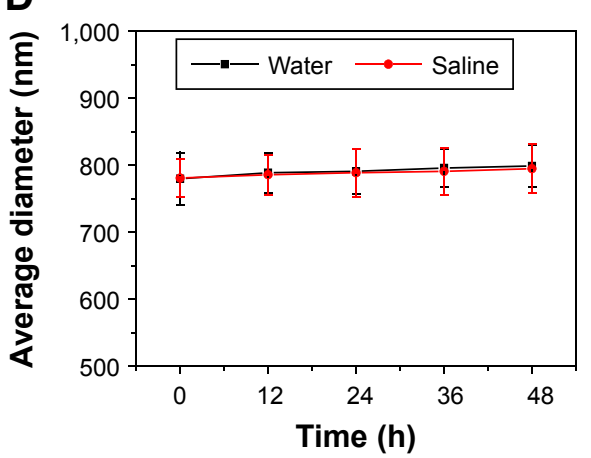

B

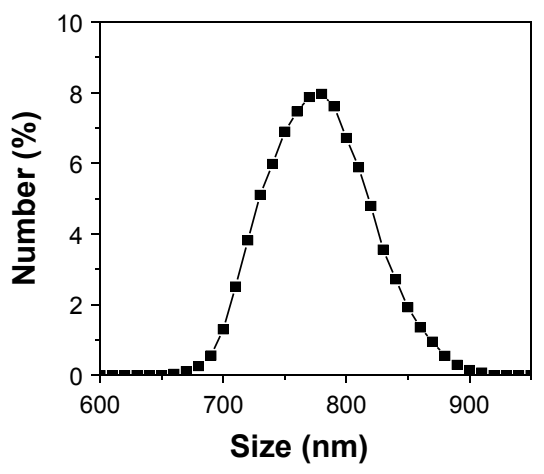

$\mathbf{E}$

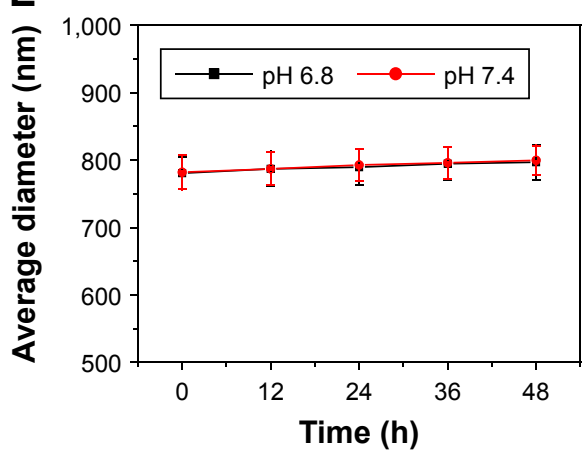

C

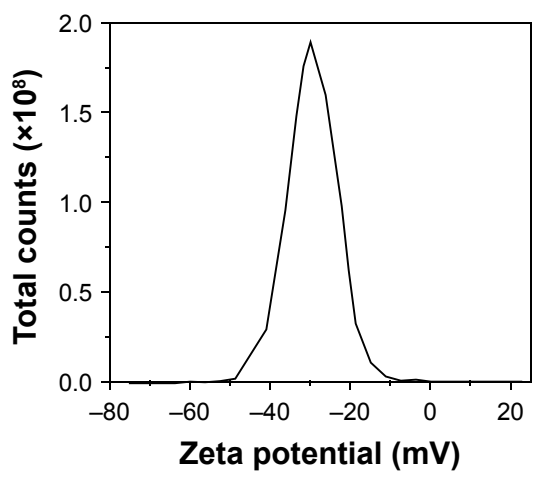

$\mathbf{F}$

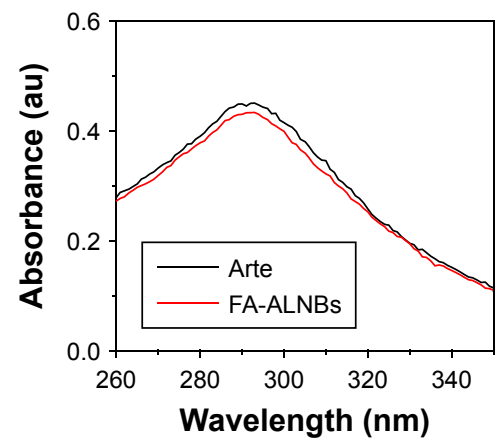

Figure 2 Morphology and stability characterization.

Notes: (A) Bright microscopy and TEM images, (B) size distribution, and (C) zeta potential of the FA-ALNBs. (D) Size changes of FA-ALNBs in water and saline, and (E) $\mathrm{pH} 6.8$ and 7.4 condition. (F) Absorbance spectra of free Arte and FA-ALNBs.

Abbreviations: Arte, artesunate; FA-ALNB, folic acid conjugated lipid nanobubbles that highly load Arte; NBs, nanobubbles; TEM, transmission electron microscopy; US, ultrasound.

negligible change in bubble size when FA-ALNBs were left to stand in water and saline, or at $\mathrm{pH}=6.8$ and $\mathrm{pH}=7.4$, for 48 hours, (Figure 2D and E), indicating the great stability of the FA-ALNBs. Their stability is due to PEG and lipid encapsulation, which can also improve the poor water solubility of loaded Arte (Figure 1A). In addition, PEG, as a surfactant, can form a water barrier on the surface of the phospholipid membrane and prolong the retention time in the blood circulation. ${ }^{22-24}$ Figure $2 \mathrm{~F}$ shows the UV-Vis spectra of FA-ALNBs, which exhibited absorbance peak similar to Arte at wavelengths of $280 \mathrm{~nm}$, demonstrating the existence of Arte in FA-ALNBs.

After calculating, the FA-ALNBs achieved 91.9\% $01.1 \%$ EE of Arte. The high Arte EE might contribute to both the optimized formulation and the preparation process, producing relatively small bubble sizes compared with those of traditional MBs. ${ }^{19}$ The drug release efficiency of FA-ALNBs with or without 1 minute US exposure by a low-frequency focus US was detected. As shown in Figure 3A, upon US irradiation with $1 \mathrm{MPa}$ US pressure and $1 \mathrm{MHz}$ frequency for 60 seconds, the drug release ratio was found to increase with increasing irradiation time. The highest Arte release rate was $41.6 \% \pm 3.5 \%$ after US irradiation for 60 seconds. This result indicated that the FA-ALNBs have a good US-triggered drug release capability. The drug release mechanism is likely due to the US-mediated FA-ALNBs destruction (Figure 1C). In addition, as shown in Figure 3B, FA-ALNBs showed intense US imaging signal compared with the control (FA-Arte lipid NPs), which does not contain gas core, indicating that the NBs endow the FA-ALNBs with enhanced US imaging capacity (Figure 1B).

In vitro cellular uptake and hemocompatibility To evaluate the cell targeting effect of obtained FA-ALNBs, FITC was used to label the ALNBs and FA-ALNBs via physical absorption. As shown in Figure 4A, much more intense FITC green fluorescence was observed in the perinuclear region of FA-ALNBs-treated cells compared with that of free FITC, ALNBs, and FA-ALNBs + FA-treated cells, indicating that a sufficient amount of FA-ALNBs enter the cells. In addition, the cellular uptake ratio of FA-ALNBs was quantified as $52.1 \% \pm 2.6 \%$ by flow cytometry, which was higher than that of free FITC, ALNBs, and FA-ALNBs + FA blocking (Figure 4B). The high cellular uptake of 
A

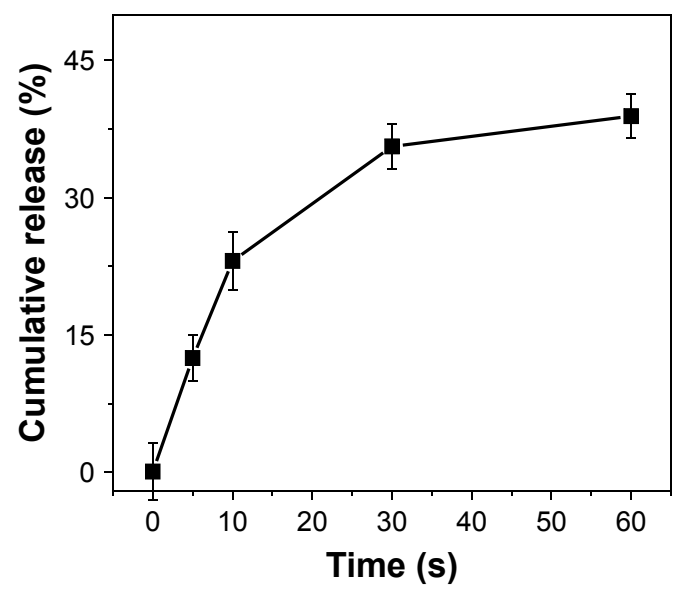

B

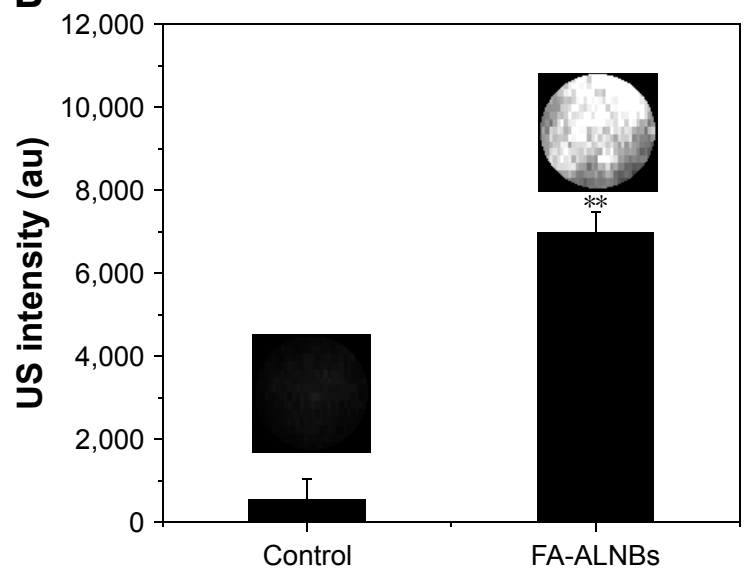

Figure 3 In vitro Arte release and US imaging.

Notes: (A) Drug cumulative release behavior under US irradiation with a frequency of I MHz over 60 seconds. (B) US images and corresponding statistical result of control (FA-Arte lipid NPs) and FA-ALNBs phantom. $* * P<0.01$ vs control group.

Abbreviations: FA-ALNB, folic acid conjugated lipid NBs that highly load Arte; NBs, nanobubbles; US, ultrasound.

A
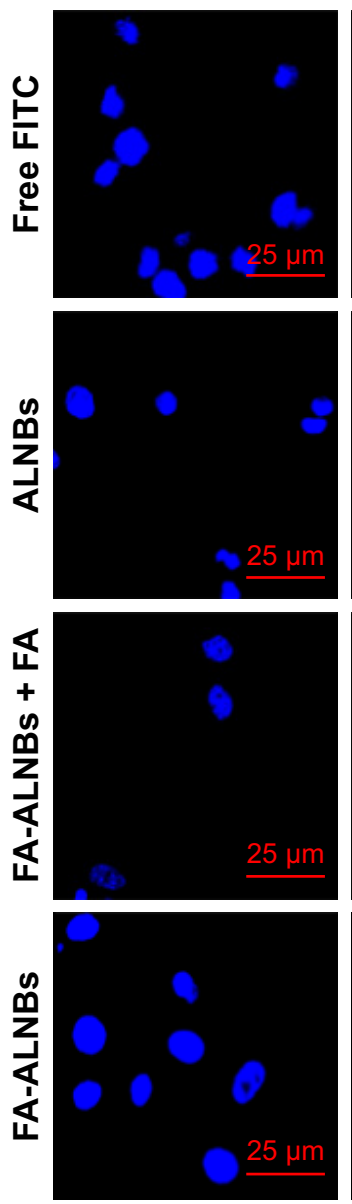

FITC
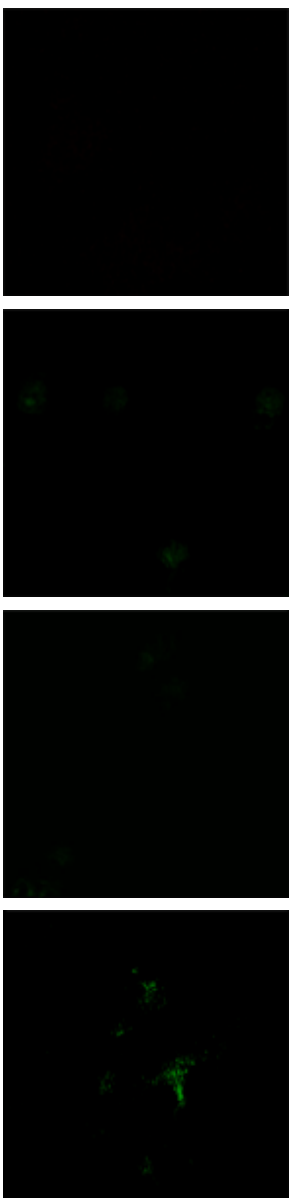
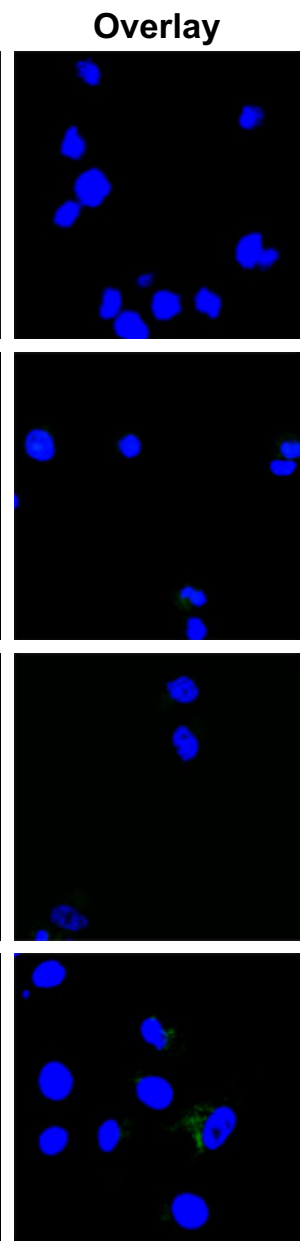

B

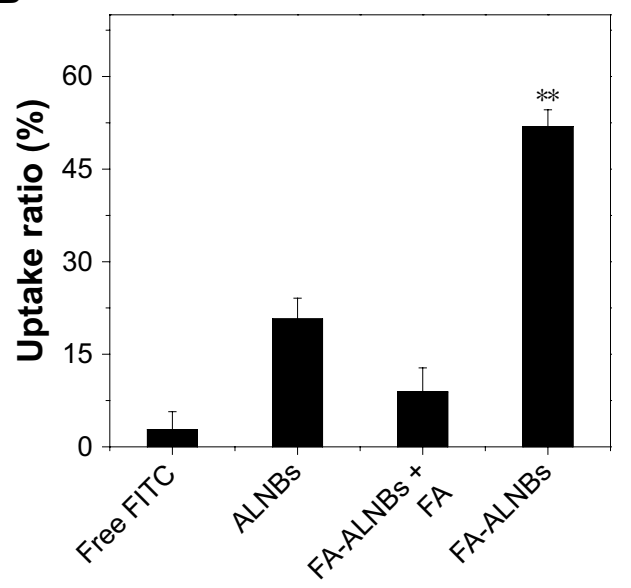

C

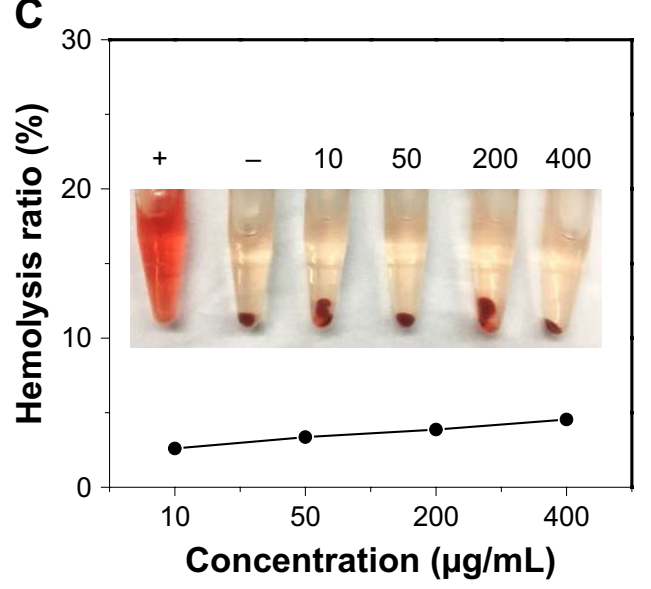

Figure 4 In vitro cellular uptake and hemocompatibility.

Notes: (A) Fluorescence images of 4 TI cells after 4 hours incubation with free FITC, FITC-labeled ALNBs and FA-ALNBs, and FITC-labeled FA-ALNBs + FA blocking. (B) Uptake ratio analysis of 4TI cells after 4 hours incubation with free FITC, FITC-labeled ALNBs and FA-ALNBs, and FITC-labeled FA-ALNBs + FA blocking using the flow cytometry. ${ }^{* * P}<0.01$ vs other tested groups. (C) Hemolysis ratio of RBCs after 3-hour incubation with FA-ALNBs at different concentrations of MBs. The inset shows the photograph of RBCs exposed to distilled water (+), PBS (-), and RILMBs-FA with different concentrations of MBs followed by centrifugation.

Abbreviations: Arte, artesunate; FA-ALNB, folic acid conjugated lipid NBs that highly load Arte; FITC, fluorescein isothiocyanate; NBs, nanobubbles; RBC, red blood cells; US, ultrasound. 

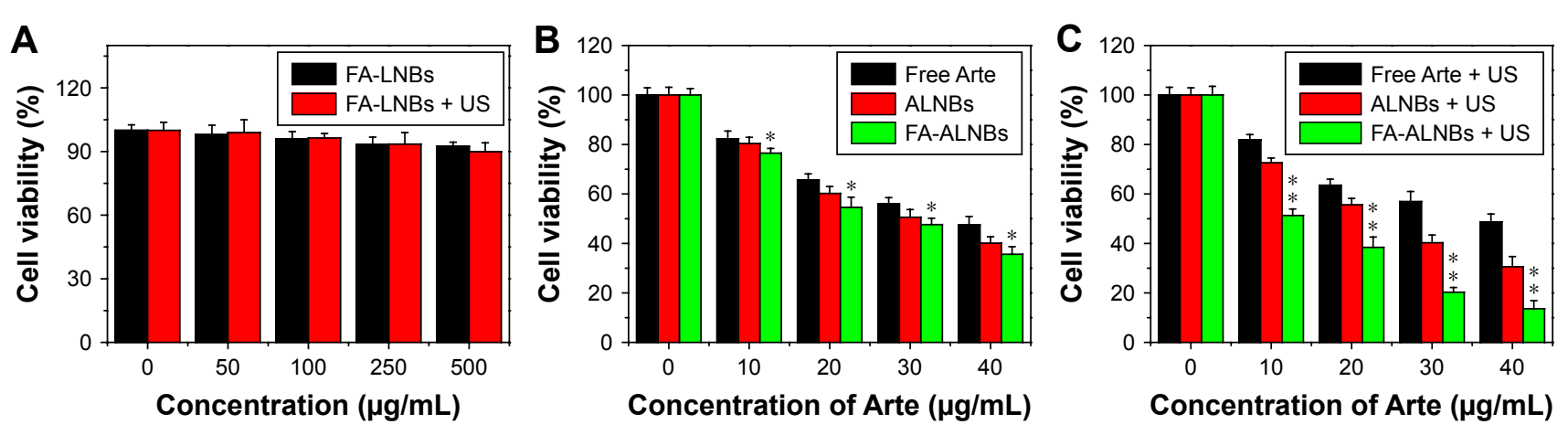

Figure 5 In vitro anticancer efficacy.

Notes: (A) In vitro cytotoxicity of different concentrations of FA-LNBs and FA-LNBs + US. (B) Cell viability of cells incubated with free Arte, ALNBs, and FA-ALNBs (at the same concentration of Arte). $* P<0.0$ I vs other tested groups. (C) Cell viability of cells incubated with free Arte + US, ALNBs + US, and FA-ALNBs + US (at the same concentration of Arte). ${ }^{* *} P<0.01$ vs other tested groups.

Abbreviations: Arte, artesunate; FA-ALNB, folic acid conjugated lipid NBs that highly load Arte; US, ultrasound; NBs, nanobubbles.

FA-ALNBs is likely due to the FA target effect via the FA receptor-mediated endocytosis pathway. ${ }^{25,26}$

In addition, as shown in Figure 4C, FA-ALNBs at a concentration range of $10-400 \mu \mathrm{g} / \mathrm{mL}$ induced a low hemolysis ratio that was similar to the negative control. This finding indicated that FA-ALNBs featured appropriate hemocompatibility characteristics.

\section{In vitro anticancer efficacy}

As shown in Figure 5A, cells treated with FA-LNBs with or without US irradiation for 24 hours exhibited no significant viability decrease, indicating no cytotoxicity of FA-LNBs and FA-LNBs + US irradiation. In addition, free Arte, ALNBs, and FA-ALNBs with the same Arte concentration (ranging between 0 and $40 \mu \mathrm{g} / \mathrm{mL}$ ) showed enhanced cell-killing ability in a dose-dependent manner (Figure 5B). Meanwhile, under 1 minute US irradiation, FA-ALNBs caused the highest cell death rate (Figure 5C). The enhanced in vitro anticancer efficacy of FA-ALNBs may be partly attributed to the efficient FA-mediated active target effect and partly to the increased drug concentration due to the US-mediated FA-ALNBs destruction. ${ }^{15}$ In addition, it was reported that under US irradiation, the NBs together with microstreaming, micro-jet, and shave wave from cavitation would further favor these US-released drugs to enter the cells. ${ }^{19}$ Compelling evidence shows that sonoporation can generate numerous nanoscale or microscale holes in the cell membrane when cells are exposed to focused US irradiation in the presence of NBs, greatly increasing cell membrane permeability..$^{27,28}$ Therefore, the simultaneous contributions of the locally released drugs and increased permeability could account for the enhanced Arte anticancer efficacy.

\section{In vivo US imaging}

As shown in Figure 6A, prior to the injection of FA-ALNBs, US images exhibited uniform hypoecho in solid tumor
A
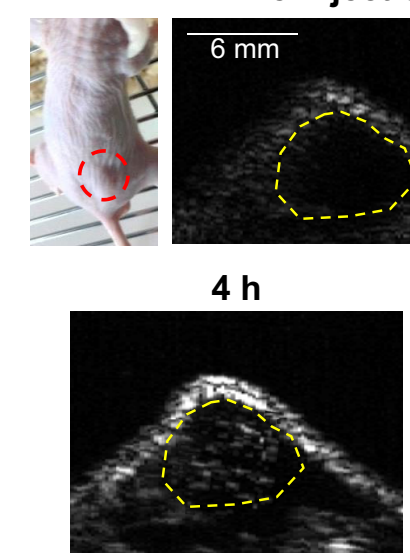

Pre-injection

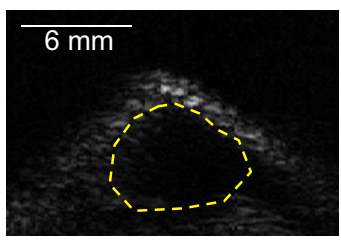

$4 \mathrm{~h}$

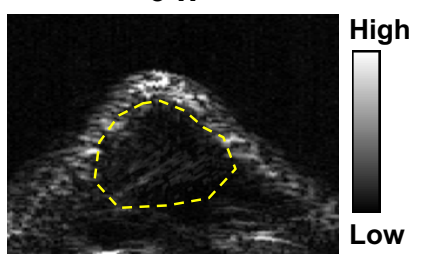

$2 \mathrm{~h}$

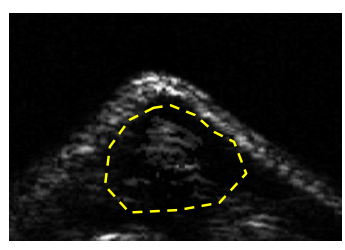

$6 \mathrm{~h}$
B

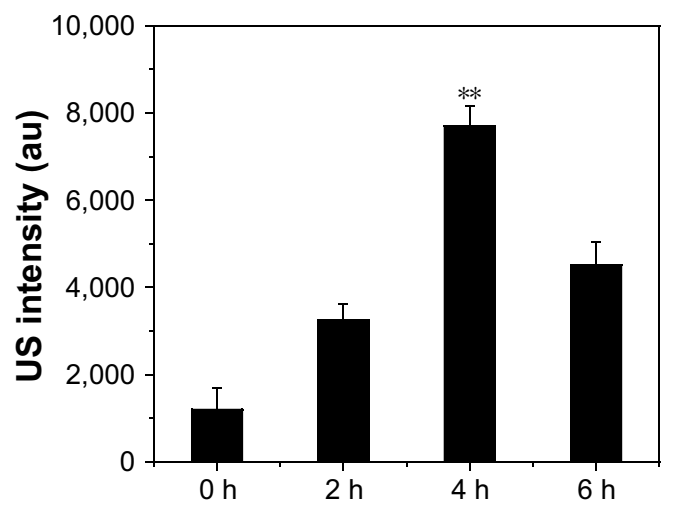

Figure 6 In vivo US imaging.

Notes: (A) US images and (B) quantitative data of tumor region at pre-injection and 2, 4, and 6 hours post injection of FA-ALNBs. $* * P<0.0$ I vs other tested time points. Red circle and yellow dotted line are tumor region.

Abbreviations: Arte, artesunate; FA-ALNB, folic acid conjugated lipid NBs that highly load Arte; NBs, nanobubbles; US, ultrasound. 
tissues. After systemic injections of FA-ALNBs, with the time increase from 2 to 6 hours, the US signal was clearly enhanced, showing a peak value at 4 hours post injection (Figure 6A). The quantitative results showed that the tumor US signal intensity of mice at 4 hours post injection of FA-ALNBs was about 7.3 folds that of pre-injection mice (Figure 6B). These results suggest that the FA-ALNBs could also act as highly sensitive US probes that enhance US imaging. This would not only facilitate the delineation of the tumor region, but would also make it possible to track Arte delivery in vivo, in a real-time manner.

\section{In vivo anticancer efficacy and toxicity evaluation}

Guided by US imaging, at 4 hours post injection of FAALNBs, 1 minute US irradiation was administered to the tumors. As expected, the tumor growth was remarkably suppressed by the FA-ALNBs + US intervention. Moreover, free Arte, US only, ALNBs, ALNBs + US, and FA-ALNBs had incomplete tumor inhibition effects, but were better than control group (Figure 7A). The greater anticancer efficacy of FA-ALNBs + US compared with that of other groups was likely due to the following reasons: 1) the increased accumulation of FA-ALNBs induced by the FA receptormediated target effect; 2) higher locally effective Arte concentration triggered by US irradiation; and 3) the US irradiation could destroy the bubble and generate physical shock to kill tumor cells. During the course of the 1-month treatment, the body weight of the mice gradually increased in all groups (Figure 7B), and the H\&E staining images
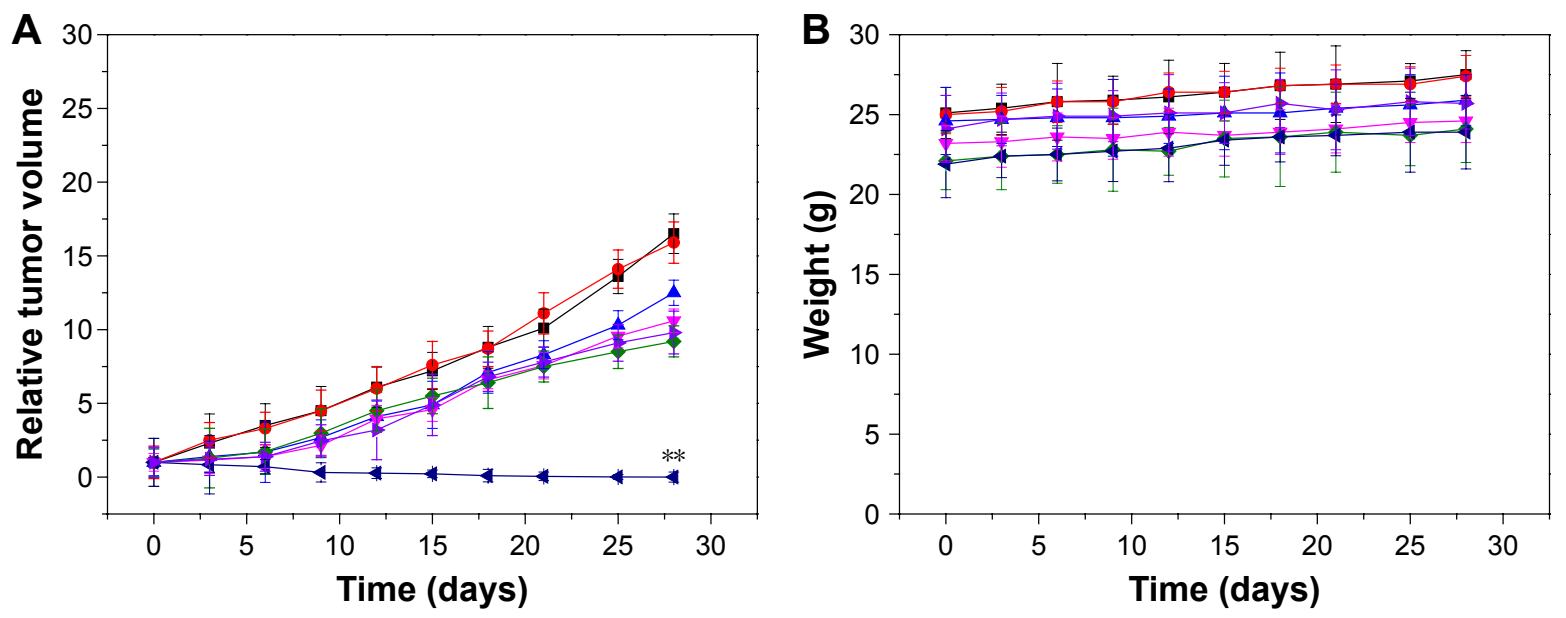

of the all treated groups showed no obvious histological lesions, which was similar to those of the control group (Figure 7C). Furthermore, the results of complete blood counts, including white blood cells, RBCs, hemoglobin, mean platelet volume, hematocrit, mean corpuscular hemoglobin concentration, and mean corpuscular volume, in FA-ALNBs-injected mice at 0,3 , and 7 days post injection were similar to values obtained pre-injection (Figure $8 \mathrm{~A}$ and B). These results indicate that the FA-ALNBs had no systemic toxicity in vivo, which is ideal for future clinical application in anticancer therapy.

\section{Conclusion}

We developed an US-triggered drug delivery system for targeted antitumor therapeutic applications. The obtained FA-ALNBs with uniform spheroidal structure and great physiological stability had high drug EE. Under US irradiation, the FA-ALNBs showed US-controllable release capability and US contrast effect. The high FA receptormediated cellular uptake was demonstrated by both confocal microscopy and flow cytometry. According to the US contrast results, maximum accumulation of FA-ALNBs was observed at 4 hours post injection of FA-ALNBs. Combined with US irradiation, FA-ALNBs showed significant anticancer efficacy both in vitro and in vivo. Moreover, the FA-ALNBs displayed no obvious systemic toxicity. Our results collectively suggest that the proposed FA-ALNBs could be a promising US-triggered Arte delivery system for imaging-guided tumor-targeted chemotherapy.

\begin{tabular}{lllll}
$\longrightarrow$ & Saline $\longrightarrow$ US $\longrightarrow$ Arte & $\longrightarrow$ & ALNBs \\
$\longrightarrow$ & ALNBs + US $\longrightarrow$ FA-ALNBs $\longrightarrow$ FA-ALNBs + US \\
\hline
\end{tabular}

Figure 7 (Continued) 

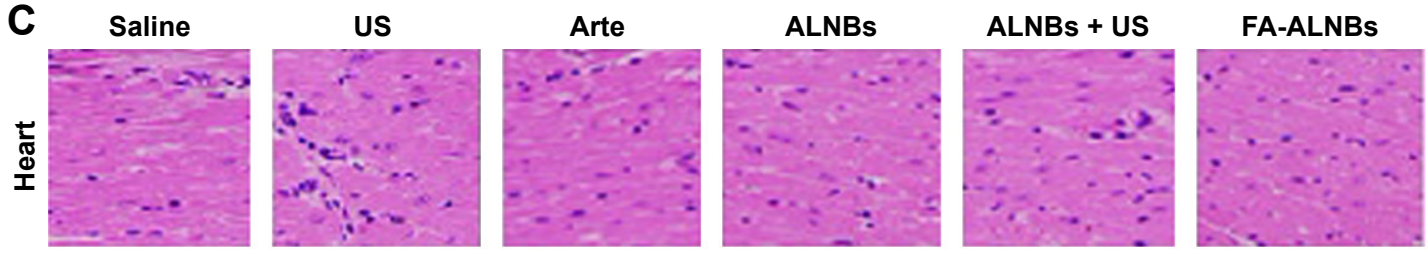

FA-ALNBs + US
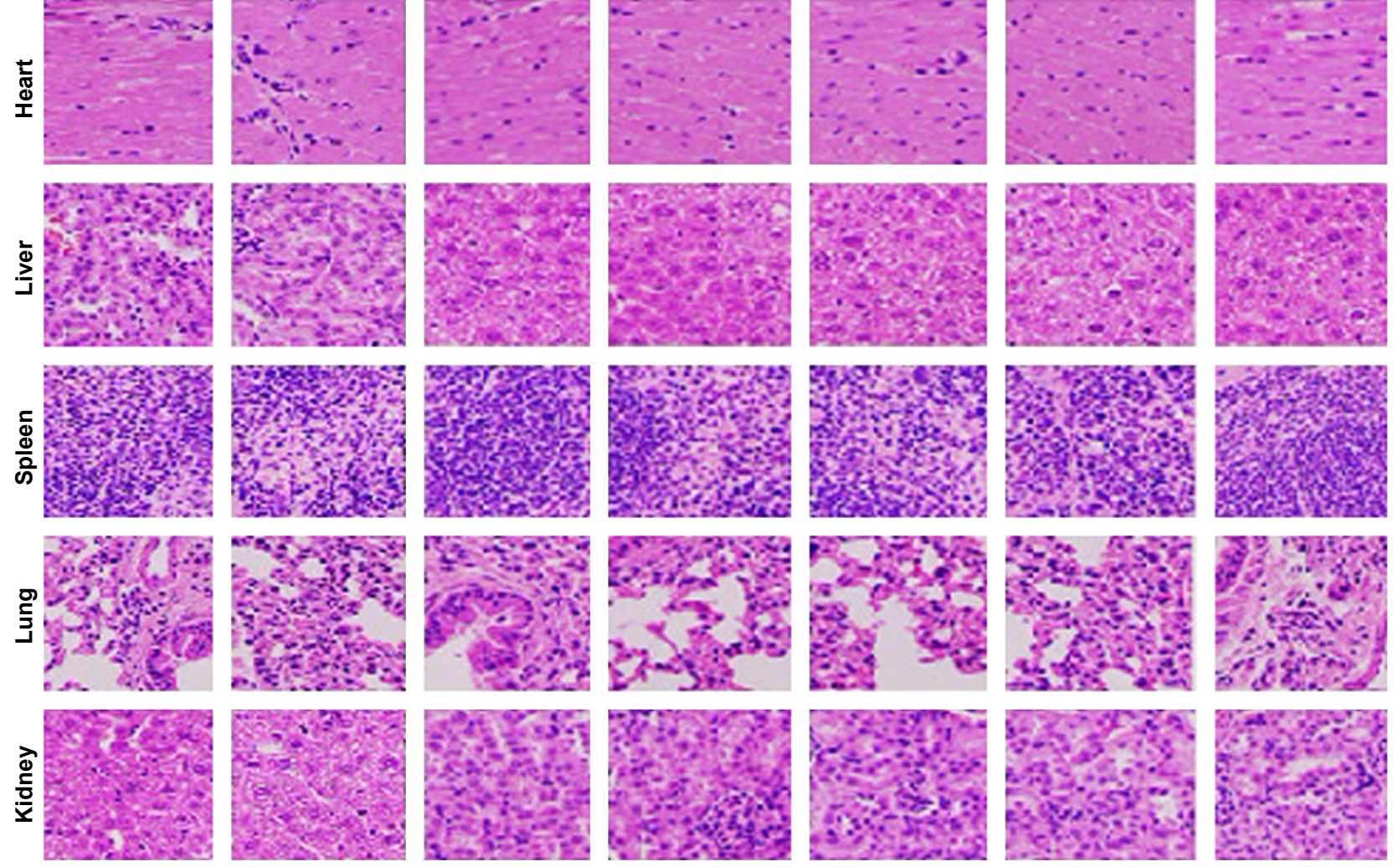

Figure 7 In vivo anticancer efficacy.

Notes: (A) Relative tumor volume and (B) body weight of tumor-bearing mice after intravenous injection with saline (control), US only, free Arte, ALNBs, ALNBs + US, FA-ALNBs, and FA-ALNBs + US. **P< $<0.01$ vs control group and other groups. (C) H\&E staining images of the major organs from the saline (control), US only, free Arte, ALNBs, ALNBs + US, FA-ALNBs, and FA-ALNBs + US treated mice (magnification: $\times 100$ ).

Abbreviations: Arte, artesunate; NBs, nanobubbles; FA-ALNB, folic acid conjugated lipid NBs that highly load Arte; US, ultrasound.

A

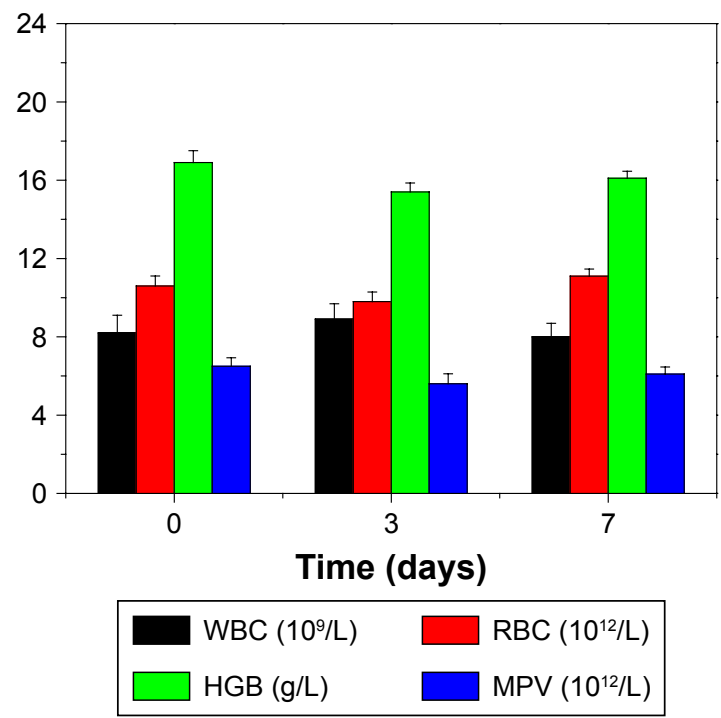

B

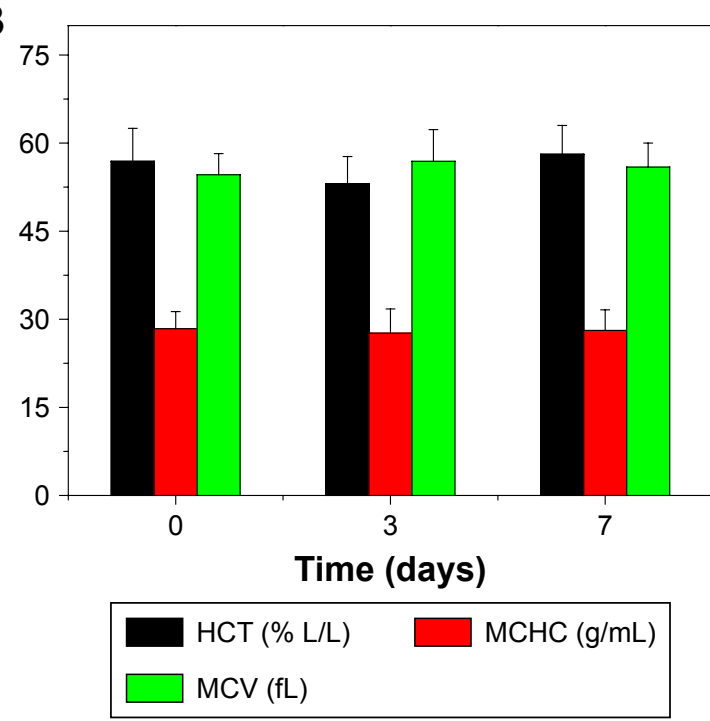

Figure 8 In vivo toxicity evaluation.

Notes: (A and B) Blood levels of WBC, RBC, HGB, MPV, HCT, MCHC, and MCV of control and FA-ALNBs-treated mice at 0, 3, and 7 days post injection.

Abbreviations: Arte, artesunate; FA, folic acid; HCT, hematocrit; HGB, hemoglobin; MCHC, mean corpuscular hemoglobin concentration; MCV, mean corpuscular volume; MPV, mean platelet volume; NBs, nanobubbles; RBC, red blood cells; US, ultrasound; WBC, white blood cells. 


\section{Acknowledgment}

The authors are grateful for the support from Gansu Provincial Hospital.

\section{Disclosure}

The authors report no conflicts of interest in this work.

\section{References}

1. Pan C, Liu Y, Zhou M, et al. Theranostic $\mathrm{pH}$-sensitive nanoparticles for highly efficient targeted delivery of doxorubicin for breast tumor treatment. Int J Nanomedicine. 2018;13:1119-1137.

2. Choudhury B, Kandimalla R, Elancheran R, Bharali R, Kotoky J. Garcinia morella fruit, a promising source of antioxidant and antiinflammatory agents induces breast cancer cell death via triggering apoptotic pathway. Biomed Pharmacother. 2018;103:562-573.

3. Weigelt B, Peterse JL, van't Veer LJ. Breast cancer metastasis: markers and models. Nat Rev Cancer. 2005;5(8):591-602.

4. Bonifácio BV, Silva PB, Ramos MA, Negri KM, Bauab TM, Chorilli M. Nanotechnology-based drug delivery systems and herbal medicines: a review. Int J Nanomedicine. 2014;9:1-15.

5. Jung IL. Soluble extract from Moringa oleifera leaves with a new anticancer activity. PLoS One. 2014;9(4):e95492.

6. Elfawal MA, Towler MJ, Reich NG, Weathers PJ, Rich SM. Dried whole-plant Artemisia annua slows evolution of malaria drug resistance and overcomes resistance to artemisinin. Proc Natl Acad Sci U S A. 2015;112(3):821-826.

7. Berte N, Lokan S, Eich M, Kim E, Kaina B. Artesunate enhances the therapeutic response of glioma cells to temozolomide by inhibition of homologous recombination and senescence. Oncotarget. 2016; 7(41):67235-67250.

8. Lian S, Shi R, Huang X, et al. Artesunate attenuates glioma proliferation, migration and invasion by affecting cellular mechanical properties. Oncol Rep. 2016;36(2):984-990.

9. Zhang P, Luo HS, Li M, Tan SY. Artesunate inhibits the growth and induces apoptosis of human gastric cancer cells by downregulating COX-2. Onco Targets Ther. 2015;8:845-854.

10. Ilamathi M, Prabu PC, Ayyappa KA, Sivaramakrishnan V. Artesunate obliterates experimental hepatocellular carcinoma in rats through suppression of IL-6-JAK-STAT signalling. Biomed Pharmacother. 2016;82:72-79.

11. Pang Y, Mai Z, Wang B, et al. Artesunate-modified nano-graphene oxide for chemo-photothermal cancer therapy. Oncotarget. 2017;8(55): 93800-93812.

12. Raza A, Ghoshal A, Chockalingam S, Ghosh SS. Connexin-43 enhances tumor suppressing activity of artesunate via gap junction-dependent as well as independent pathways in human breast cancer cells. Sci Rep. 2017;7(1):7580.
13. Song R, Peng C, Xu X, et al. Controllable formation of monodisperse polymer microbubbles as ultrasound contrast agents. ACS Appl Mater Interfaces. 2018;10(17):14312-14320.

14. Mai L, Yao A, Li J, et al. Cyanine 5.5 conjugated nanobubbles as a tumor selective contrast agent for dual ultrasound-fluorescence imaging in a mouse model. PLoS One. 2013;8(4):e61224.

15. Lv Y, Cao Y, Li P, et al. Ultrasound-triggered destruction of folatefunctionalized mesoporous silica nanoparticle-loaded microbubble for targeted tumor therapy. Adv Healthc Mater. 2017;6(18):1700354.

16. Deng Z, Yan F, Jin Q, et al. Reversal of multidrug resistance phenotype in human breast cancer cells using doxorubicin-liposome-microbubble complexes assisted by ultrasound. J Control Release. 2014;174(1): 109-116.

17. Huang HY, Hu SH, Hung SY, et al. SPIO nanoparticle-stabilized PAA-F127 thermosensitive nanobubbles with MR/US dual-modality imaging and HIFU-triggered drug release for magnetically guided in vivo tumor therapy. J Control Release. 2013;172(1):118-127.

18. Hernot S, Klibanov AL. Microbubbles in ultrasound-triggered drug and gene delivery. Adv Drug Deliv Rev. 2008;60(10):1153-1166.

19. Zhu X, Guo J, He C, et al. Ultrasound triggered image-guided drug delivery to inhibit vascular reconstruction via paclitaxel-loaded microbubbles. Sci Rep. 2016;6(1):21683.

20. Huang WC, Chiang WH, Cheng YH, et al. Tumortropic monocytemediated delivery of echogenic polymer bubbles and therapeutic vesicles for chemotherapy of tumor hypoxia. Biomaterials. 2015;71:71-83.

21. Yan F, Li L, Deng Z, et al. Paclitaxel-liposome-microbubble complexes as ultrasound-triggered therapeutic drug delivery carriers. J Control Release. 2013;166(3):246-255.

22. Pasut G, Paolino D, Celia C, et al. Polyethylene glycol (PEG)-dendron phospholipids as innovative constructs for the preparation of super stealth liposomes for anticancer therapy. J Control Release. 2015;199(1): 106-113.

23. Nag M, Gajbhiye V, Kesharwani P, Jain NK. Transferrin functionalized chitosan-PEG nanoparticles for targeted delivery of paclitaxel to cancer cells. Colloids Surf B Biointerfaces. 2016;148:363-370.

24. Zulfiqar B, Mahroo A, Nasir K, et al. Nanomedicine and cancer immunotherapy: focus on indoleamine 2,3-dioxygenase inhibitors. Oncotargets Ther. 2017;10:463-476.

25. Dai L, Liu J, Luo Z, Li M, Cai K. Tumor therapy: targeted drug delivery systems. J Mater Chem B. 2016;4(42):6758-6772.

26. Lee JJ, Saiful Yazan L, Che Abdullah CA. A review on current nanomaterials and their drug conjugate for targeted breast cancer treatment. Int J Nanomedicine. 2017;12:2373-2384.

27. Tu J, Zhang D. The impacts of microbubble-induced sonoporation on cell membrane permeability and cytoskeleton arrangement. Ultrasound Med Biol. 2017;43:S234.

28. Chen CS, Zhao Q, Qian S, et al. Ultrasound-guided RNA interference targeting HIF-1 alpha improves the effects of transarterial chemoembolization in rat liver tumors. OncoTargets Ther. 2015;8:3539-3548.
OncoTargets and Therapy

\section{Publish your work in this journal}

OncoTargets and Therapy is an international, peer-reviewed, open access journal focusing on the pathological basis of all cancers, potential targets for therapy and treatment protocols employed to improve the management of cancer patients. The journal also focuses on the impact of management programs and new therapeutic agents and protocols on

\section{Dovepress}

patient perspectives such as quality of life, adherence and satisfaction. The manuscript management system is completely online and includes a very quick and fair peer-review system, which is all easy to use. Visit http://www.dovepress.com/testimonials.php to read real quotes from published authors. 